\title{
Technical standards and guidelines for myotonic dystrophy type 1 testing
}

\author{
Thomas W. Prior, PhD; on behalf of the American College of Medical Genetics (ACMG) \\ Laboratory Quality Assurance Committee
}

\begin{abstract}
Disclaimer: These standards and guidelines are designed primarily as an educational resource for clinical laboratory geneticists to help them provide quality clinical laboratory genetic services. Adherence to these standards and guidelines does not necessarily ensure a successful medical outcome. These standards and guidelines should not be considered inclusive of all proper procedures and tests or exclusive of other procedures and tests that are reasonably directed to obtaining the same results. In determining the propriety of any specific procedure or test, the clinical molecular geneticist should apply his or her own professional judgment to the specific clinical circumstances presented by the individual patient or specimen. It may be prudent, however, to document in the laboratory record the rationale for any significant deviation from these standards and guidelines.
\end{abstract}

\begin{abstract}
Myotonic dystrophy type 1 is an autosomal dominant multisystem condition. Myotonic dystrophy type 1 is the result of an unstable CTG expansion in the $3^{\prime}$-untranslated region of the myotonic dystrophy protein kinase gene. The age of onset and the severity of the phenotype are roughly correlated with the size of the CTG expansion. The combination of Southern transfer and polymerase chain reaction provides an accurate means of identifying patients affected by myotonic dystrophy type 1 . This document follows the outline format of the general Standards and Guidelines for Clinical Genetics Laboratories. It is designed to be a checklist for genetic testing professionals who are already familiar with the disease and the methods of analysis. Genet Med 2009:11(7):552-555.
\end{abstract}

Key Words: myotonic dystrophy type 1, CTG repeats, genetic testing, genotype, phenotype

$\mathrm{D}$ isease-specific statements are intended to augment the current general American College of Medical Genetics (ACMG) Standards and Guidelines for Clinical Genetic Laboratories. Individual laboratories are responsible for meeting the CLIA/CAP quality assurance standards with respect to appropriate sample documentation, assay validation, general proficiency, and quality control measures.

\section{BACKGROUND ON MYOTONIC DYSTROPHY TYPE 1}

Gene symbol/chromosome locus: Myotonic dystrophy protein kinase gene at chromosome 19q13.3. OMIM number: 160900.

From the Department of Pathology, Ohio State University, Columbus, Ohio. Thomas W. Prior, PhD, Department of Pathology, The Ohio State University, 125 Hamilton Hall, 1645 Neil Avenue, Columbus, OH 43210. E-mail: thomas.prior@osumc.edu.

Disclosure: The author declares no conflict of interest

Submitted for publication April 27, 2009.

Accepted for publication April 27, 2009.

Published online ahead of print June 19, 2009.

DOI: 10.1097/GIM.0b013e3181abce0f
Brief clinical description: Myotonic dystrophy type 1 (DM1) is an adult/congenital-onset multisystem disorder characterized by progressive muscle weakness, myotonia, intellectual impairment, cataracts, cardiac arrhythmias, respiratory insufficiency, hypogonadism, and endocrine disturbances. ${ }^{1}$ The diagnosis can be problematic because of the wide range and severity of symptoms and often affected individuals will already have children before they have been diagnosed. The rare severe congenital form of the disorder results in mental retardation, respiratory distress, hypotonia, and in many cases death shortly after birth due to respiratory complications. Those who survive the neonatal period initially follow a static course, eventually learning to walk but with significant mental retardation. The congenital form is most often observed in the offspring of women who are themselves affected, although the disease in a mother may not be diagnosed until after the birth of a congenitally affected child. ${ }^{1}$ For more information, see the online Gene Clinics profile at www.geneclinics.org.

Mode of inheritance: Inheritance is autosomal dominant with incomplete penetrance and variable expressivity.

Gene description/normal gene product: The myotonic dystrophy protein kinase $(D M P K)$ gene is approximately 12 to 14 $\mathrm{kb}$ long and contains 14 or 15 exons, as defined by comparisons between cDNAs and genomic sequence. Transcription of the gene results in the production of several alternatively spliced forms, all of which contain the kinase catalytic domain. There is a differential expression of the alternatively spliced isoforms in different tissues. The CTG repeat occurs in the $3^{\prime}$ untranslated region (UTR) corresponding to the last exon. The gene encodes a serine-threonine kinase, which is expressed in all tissues affected in DM1.

The DM1 mutation: DMPK is the only known gene associated with DM1. The DM1 mutation involves an expanded CTG trinucleotide repeat located at the $3^{\prime}$ UTR of the DMPK gene. ${ }^{2-4}$ The number of CTG triplets varies in the normal population from 5 to 34, and within this range the alleles are stably transmitted. Individuals with 35 to 49 repeats (premutation alleles) do not have symptoms but their children are at an increased risk of inheriting larger repeats and having symptoms. When the repeat length exceeds 50 CTGs (in some patients up 
to several thousands), the allele becomes unstable and results in the DM1 phenotype.

Genotype/phenotype association: There is a significant correlation between the CTG repeat size and the age-of-onset and clinical severity. 5,6 Mildly affected patients have 50 to 100 repeats, and these patients may only report cataracts and/or mild myotonia. More classically affected patients have 100 to 1000 repeats and congenital cases often have 1000 to 6000 repeats. However, there is dynamic somatic mosaicism of the CTG repeat biased toward continuous expansion throughout the lifetime of an affected individual. Furthermore, there is an overlap in repeats sizes in patients with differing severity of the disease. The trinucleotide is mitotically and meiotically unstable with a bias toward length increase in the next generation accounting for the phenomenon of "anticipation" (increasing severity in successive generations of the same family, with earlier age of onset). Although repeat expansions occur through both maternal and paternal transmissions, the larger repeat expansions observed in congenital cases are almost exclusively due to maternal transmissions. Several cases of reverse mutations (i.e., contraction to normal range of repeats) have also been reported in DM, whereby there is a spontaneous reversion of a deleterious mutation on transmission to an unaffected offspring. The mechanism for the DM1 reverse mutations remains unknown. A gene conversion mechanism, whereby the normal parental allele replaces the expanded allele or a double recombination event leading to a disruption of the CTG repeats have been proposed as possible mechanisms for spontaneous contractions. The reversions may provide an explanation of the nonpenetrance observed in some DM1 families. Therefore, challenges that call for caution in genetic counseling of patients and families affected by DM1 include extreme clinical variability, somatic mosaicism, anticipation, influence of gender of the affected patient, and nonpenetrance due to reversions.

Mutational mechanism: The CTG repeat is located within the 3' UTR of a gene that encodes a protein kinase, named myotonin protein kinase. Because the repeat is not in the protein coding portion (i.e., the exons), the molecular mechanism by which the mutation exerts its dominant expression is difficult to explain. It has been suggested that the myotonin kinase mRNA with long CUG repeats, and not the protein, result in a gain-offunction RNA pathogenesis. ${ }^{7-9}$ Novel RNA binding proteins that specifically bind to CUG repeats may be depleted by excessive CUG repeats in the DM1 transcripts. Muscleblindlike protein-1 and CUG-binding protein-1 are two RNA binding proteins proposed to be involved in the pathogenesis. The depletion of these CUG-binding proteins has been shown to cause splicing alterations of the chloride channel-1 gene and the insulin receptor genes resulting in myotonia and insulin resistance, phenotypes that are related to the clinical features of DM1.

Listing of mutations: Mutations at locations other than the CTG expansion at the $3^{\prime}$ UTR of the gene have not been described in DM1.

Ethnic association of DM1 mutation: The prevalence of DM1 ranges from $1 / 100,000$ in some areas of Japan to $1 / 10,000$ in Iceland, 1/18,000 among Asians, and very low reported incidence among African blacks. The estimated worldwide prevalence of DM1 is $1 / 20,000$.

\section{TESTING CONSIDERATIONS}

Analytical sensitivity and analytical specificity: CTG-repeat expansion mutations account for $>99 \%$ of cases of DM1. Therefore, the analytical sensitivity and specificity of tests that effectively detect and measure the CTG repeat in the $3^{\prime}$ UTR of the DMPK1 gene approaches $100 \%$. However, the DM1 mutation can be carried by asymptomatic or minimally affected individuals who have relatively small expansions, in the range of approximately 50 to 100 repeats. Therefore, factors such as age, family history, penetrance, and variable expressivity preclude an accurate determination of the clinical sensitivity and specificity of the test in individuals with one or both alleles in the range of 50 to 100 repeats. For CTG repeats larger than this range, the clinical sensitivity and specificity are high and expected to approach $100 \%$. Allele sizes of 35 to 49 CTG repeats (premutation alleles) are rare and have been mostly ascertained through their symptomatic offspring, which expanded $>50$ repeats. Myotonic dystrophy type 2 (DM2, MIM 602668) is an autosomal dominant disorder, previously termed proximal myotonic myopathy, due to a CCTG expansion located in intron 1 of the zinc finger protein 9 gene on chromosome $3 \mathrm{q} 21 .{ }^{10}$ Individuals affected with DM2 also have a complex clinical presentation that is similar to DM1 including myotonia, cardiac involvement, cataracts, hypogonadism, insulin insensitivity, and histological abnormalities in skeletal muscle. However, patients with DM2 can often be distinguished from the majority of cases from DM1 by a more proximal muscle weakness and sparing of facial muscles. Further distinguishing characteristic features of DM2 include muscle pain, absence of congenital cases, and milder course of the disease without significant mental involvement.

Indications for genetic testing: This test is often used for symptomatic confirmatory diagnostic testing and predictive testing, after the identification of the mutation in an affected family member. The test is also useful for prenatal diagnosis for at-risk pregnancies after ultrasound evidence of fetal hypotonia, reduced fetal movements, positional abnormalities, and/or polyhydramnios. The testing is also extremely helpful in identifying individuals who are asymptomatic or exhibit equivocal symptoms, such as cataracts. No new mutations have been described in DM, which is consistent with the linkage disequilibrium data. To account for the maintenance of the mutation in the population, it was proposed that there is a high incidence of minimally expanded alleles in DM1 families, which produce few symptoms and are stably transmitted over several generations. Therefore, for counseling purposes, it becomes important to identify which side of the family the mutation is segregating. When comparing unrelated affected individuals with small to moderate differences in repeat sizes, it is generally difficult to accurately predict the severity of the disease in each case. This is due to the overlap of triplet repeat size in patients with differing severity of the disease and sometimes lack of a correlation between organ involvement and repeat size. ${ }^{11}$ However, when a child has a significant increase in allele size when compared with the parent, it is almost certain that there will be an earlier age of onset and more severe disease. ${ }^{5}$ It is strongly recommended that genetic counseling be offered to not only the affected patient but also to other at-risk interested family members. This test can be used for prenatal diagnosis in both amniotic fluid cells and chorionic villus samples (CVS). Maternal cell contamination studies should be performed on every prenatal sample to confirm the fetal origin of the sample being tested.

\section{GUIDELINES}

\section{Definition of normal and mutation category}

Normal alleles have a range of $\sim 5$ to 34 . These alleles are not pathologic and segregate as stable polymorphic repeats. The 
distribution of normal alleles is not random. A trimodal distribution is observed in European populations and in white Americans, with the most frequently occurring allele being five repeats. The second mode consists of three major alleles of copy numbers 11,12 , and 13 and a minor allele of 14 repeats. The final mode has no clear peak but represents alleles of 19 repeats or more. ${ }^{12}$

Premutation alleles have a range of $\sim 35$ to 49 . DM1 premutation alleles are often identified in distant relatives of DM1 probands. These alleles are not associated with a clinical phenotype in the carrier but are unstable and liable to expand in succeeding generations.

Full penetrant alleles are greater than $>50$. These repeats are unstable and are associated with disease manifestations.

Mosaicism: Patients with DM1 often show marked somatic mosaicism of the CTG repeat. The expansions often give a diffuse or smeared appearance on Southern blotting. Heterogeneity within and differences in average repeat lengths between tissues in the same individual has been confirmed. Somatic mosaicism in various tissues may be a probable explanation for the lack of a correlation between the repeat size of DNA from blood lymphocytes and clinical symptoms in some cases. In one study, CTG expansions were 2- to 13-fold greater in DNA isolated from skeletal muscle than in DNA isolated from leukocytes in 10 of 11 patients with DM1. ${ }^{11}$

\section{METHODOLOGICAL CONSIDERATIONS}

Individual US laboratories offering molecular diagnostic testing for DM1 should be in compliance with all federal and state regulations relevant to clinical laboratory operations. This includes meeting all Clinical Laboratory Improvement Amendments/College of American Pathologists quality control requirements. In addition, all laboratories should be active participants in annual DM1 proficiency testing challenges. All methodological applications should also be in compliance with the current Standards and Guideline for Clinical Genetics Laboratories developed by the Laboratory Practice Committee of the ACMG. Non-US laboratories should be similarly compliant with their individual countries statutory regulations governing oversight of clinical laboratories.

The direct DNA tests have reduced the number of invasive (muscle biopsy) and noninvasive (electromyography) diagnostic techniques for the diagnosis of DM1. The combination of Southern transfer and polymerase chain reaction (PCR) can detect all DM1 mutations. ${ }^{13}$ The majority of clinically significant mutations can be identified by Southern analysis; however, for small amplifications ( $<100$ repeats) PCR is essential. Although the PCR test is less expensive and faster than the Southern blot, longer repeats are often not reliably amplified. All general guidelines for Southern transfer and PCR in the ACMG Standards and Guidelines apply. The following additional details are specific for DM1 molecular testing.

\section{Southern blots}

Probes: Several probes are available for hybridization including PGB2.6, pMDY1, cDNA25, and p5B1.4.3,4,14,15 Several of the probes will detect not only the DM1 mutation but also an Alu repeat insertion/deletion polymorphism within the restriction fragment. It has been shown that the insertion allele is almost in complete linkage disequilibrium with the mutation suggesting that the disease appeared to be the result of one or a few ancestral mutations.

Restriction enzymes: For the best resolution of smaller expansions, a restriction enzyme should be chosen which allows

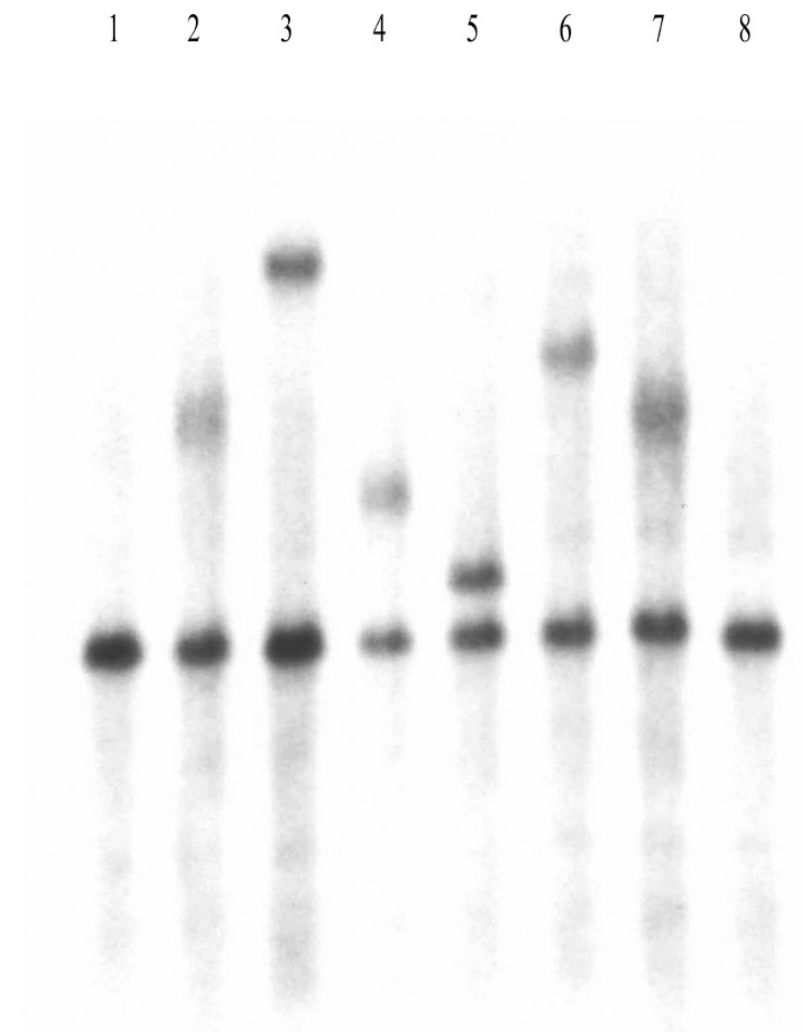

Fig. 1. Southern blot detection of the CTG expansion in the DM1 gene. HindllI-Bgll digested genomic DNA probed with pMDY1. Samples in Lanes 1 and 8 are unaffected controls. Samples from patients with DM1 (Lanes 2-7) show an expanded fragment representing the mutant allele. Normal allele is $2.2 \mathrm{~kb}$.

the probe to hybridize to a smaller fragment. By reducing the size of the restriction fragment, resolution is improved and expansions as small as 100 repeats can easily be detected. Figure 1 shows a Southern analysis with DNA, from several patients, digested with HindIII and $B g l$ I run on $0.8 \%$ agarose gel and blotted on a nylon membrane. Increasing the electrophoretic migration will also improve the detection of smaller expansions. The background may interfere with the detection of the larger expanded alleles because the expanded alleles often appear as diffuse smears due to the somatic instability of the mutation. The efficiency of the detection of these somatically variable expansions can be increased by the application of relatively infrequent cutting enzyme that will generate a larger restriction fragment containing the expansion. EcoRI cuts a large 9 to 10 $\mathrm{kb}$ fragment and HindIII cuts 8.5 to $9.5 \mathrm{~kb}$ fragment in the DM1 gene. The larger fragment lengths will reduce the smearing effect of the somatic variability of the unstable repeats. Decreasing the electrophoretic migration will lead to better band definition resulting in an improved detection of larger somatic mosaic expansions as distinct bands instead of diffuse smears. Therefore, the most efficient approach for identifying expansions may be a combination of different restriction enzymes and variation in electrophoretic duration. ${ }^{16,17}$

Expansion sizes can be estimated from Southern transfers by using a standard ladder such as lambda HindIII fragments or a set of chosen controls. 


\section{PCR methods}

Several sets of primers, PCR conditions, amplicon separation, and detection techniques have been published. Regardless of the particular PCR-based strategy selected, it is important that assay conditions and post-PCR analyses be optimized to ensure for the accurate and unambiguous sizing of repeat lengths.

PCR can be used to detect smaller expansions, usually observed in milder cases, which are difficult to resolve by Southern blotting. However, longer repeats are not reliably detected by PCR, and therefore the method is not suitable to make a direct diagnosis in many cases.

The PCR test can be useful in excluding DM1, when individuals demonstrate two different normal size alleles. When two normal alleles are identified, the DM1 diagnosis can be excluded and the Southern transfer testing is not necessary. However, since the heterozygosity frequency for the CTG repeats is $\sim 75 \%$ in the normal population, $\sim 25 \%$ of unaffected individuals will be homozygous for a given normal allele. Therefore, the presence of a single PCR band does not confirm a diagnosis of DM1. All single bands require a Southern confirmation.

Accurate sizing of repeat lengths should be empirically determined by comparison with appropriate external or internal standards. These could include, but are not limited to, (M13) sequencing ladders, cloned reference standards, and appropriate normal and abnormal patient controls whose sizes have been independently verified.

For each analysis, appropriate controls that include a range of CTG sizes should be used. It is the responsibility of the laboratory to empirically determine the detection limits for their assays.

\section{INTERPRETATIONS}

Elements considered essential to the reporting of clinical test results are described in detail in the current ACMG Standards and Guidelines for Clinical Genetics Laboratories. The following additional elements must also be included in the reporting of the DM1 genotype.

The methodology used to assign the genotype. If PCR methodology was used, then a description of the primer pair(s) should be included as well as the method of amplicon separation and detection. If a Southern transfer was required, the restriction enzyme(s) and probe(s) should be identified. Each report must state the CTG repeat length categories and descriptors currently used in clinical practice and each reportable genotype should be classified and interpreted using these categorical definitions.

The normal, premutation, and affected repeat ranges should be clearly stated in the report. Each report must include the CTG repeat numbers of both alleles with the precision of sizing fulfilling the criteria recommended by the CAP/ACMG Biochemical and Molecular Genetics Resource Committee. For large alleles determined by Southern, qualifying terms such as "approximately" or "estimated" can be used but should not be written as to create any unnecessary interpretive ambiguity. All positive results should state that genetic counseling is indicated and testing is available for other at-risk family members.

Informed consent and the usual caveats should be addressed including paternity issues, possible diagnostic errors due to sample mix-ups and genotype errors due the presence of rare polymorphisms.
The following statement must be included on the report. "This test was developed and its performance characteristics determined by this laboratory. It has not been cleared or approved by the US Food and Drug Administration. The Food and Drug Administration has determined that such clearance or approval is not necessary. This analysis is used for clinical purposes. It should not be regarded as investigational or for research."

The following alternative diagnosis may be included: Proximal DM2 will not be detected by this test.

Comments on phenotype, if included, should be abstract rather case specific. The following concepts apply.

For asymptomatic testing, the priori risk of inheriting the DM1 mutation is modified by DM1 mutation analysis. However, predictions, from the repeat size, regarding degree of severity or age onset should not be included in the report.

For prenatal diagnosis, due to the overlapping ranges and uncertainty regarding somatic mosaicism and in utero instability of the expanded CTG repeat, it is not possible to predict whether the fetus will have congenital or adult-onset DM1.

\section{REFERENCES}

1. Harper PS, editor. Myotonic dystrophy, 2nd ed. Philadelphia: W.B. Saunders, 1989

2. Brook JD, McCurrach ME, Harley HG, et al. Molecular basis of myotonic dystrophy: expansion of a trinucleotide (CTG) repeat at the $3^{\prime}$ end of a transcript encoding a protein kinase family member. Cell 1992;68:799-808.

3. Fu YH, Pizzuti A, Fenwick RG Jr, King J, et al. An unstable triplet repeat in a gene related to myotonic muscular dystrophy. Science 1992;255:12561258

4. Mahadevan M, Tsilfidis C, Sabourin L, et al. Myotonic dystrophy mutation: an unstable CTG repeat in the $3^{\prime}$ untranslated region of the gene. Science 1992;255:1253-1255.

5. Redman JB, Fenwick RG Jr, Fu YH, Pizzuti A, Caskey CT. Relationship between parental trinucleotide GCT repeat length and severity of myotonic dystrophy in offspring. JAMA 1993;269:1960-1965.

6. Tsilfidis C, MacKenzie AE, Mettler G, Barceló J, Korneluk RG. Correlation between $\mathrm{CTG}$ trinucleotide repeat length and frequency of severe congenital myotonic dystrophy. Nat Genet 1992;1:192-195.

7. Ranum LP, Day JW. Myotonic dystrophy: RNA pathogenesis comes into focus. Am J Hum Genet 2004;74:793-804.

8. Charlet-BN, Savkur RS, Singh G, Philips AV, Grice EA, Cooper TA. Loss of the muscle-specific chloride channel in type 1 myotonic dystrophy due to misregulated alternative splicing. Mol Cell 2002;10:45-53.

9. Savkur RS, Philips AV, Cooper TA. Aberrant regulation of insulin receptor alternative splicing is associated with insulin resistance in myotonic dystrophy. Nat Genet 2001;29:40-47.

10. Liquori CL, Ricker K, Moseley ML, et al. Myotonic dystrophy type 2 caused by a CCTG expansion in intron 1 of ZNF9. Science 2001;293:864-867.

11. Thorton CA, Johnson K, Moxley RT III. Myotonic dystrophy patients have larger CTG expansions in skeletal muscle than in leukocytes. Ann Neurol 1994;35:104-107.

12. Martorell L, Monckton DG, Sanchez A, Lopez de Munain A, Baiget M. Frequency and stability of the myotonic dystrophy type 1 premutation. Neurology 2001;56:328-335

13. International Myotonic Dystrophy Consortium (IDMC). New nomenclature and DNA testing guidelines for myotonic dystrophy type I (DM1). Neurology 2000;54:1218-1221.

14. Buxton J, Shelbourne P, Davie J, et al. Detection of an unstable fragment of DNA specific to individuals with myotonic dystrophy. Nature 1992;355: 547-548.

15. Shelbourne P, Davies J, Buxton J, Anvret M, et al. Direct diagnosis of myotonic dystrophy with a disease-specific DNA marker. $N$ Engl J Med 1993;328:471-475.

16. Petronis A, Heng HH, Tatuch Y, et al. Direct detection of expanded trinucleotide repeats using PCR and DNA hybridization techniques. Am J Med Genet 1996;67:85-91.

17. Guida M, Marger RS, Papp AC, et al. A molecular protocol for diagnosing myotonic dystrophy. Clin Chem 1995;41:69-72. 